\title{
Promoting Ubiquity and Interoperability among Health Information Systems Using an SOA Based Architecture
}

\author{
Gonçalo Jorge Rodrigues Plácido, Carlos Rompante Cunha and Elisabete Paulo Morais
}

Polytechnic Institute of Bragança - EsACT, Rua João Sarmento Pimentel, Apartado, Mirandela, Portugal

\begin{abstract}
Health information systems are of extreme importance and they became an intrinsic part of the healthcare sector. However, in today's molds and with the advent of the Internet and mobile devices, a paradigm shift, from the current isolated systems to interoperable distributed systems, that take advantage of ubiquitous computing, is needed. In critical situations, such as decision making in healthcare, it is necessary to have access to all of the patient's information; for the information must be reliable and must be accessed in an easy and fast way. The present work proposes an architecture that aims to answer the needs of interoperability between heterogeneous health information systems and the need for ubiquity of medical information. A prototype was developed that tries to provide interoperability through a service-oriented architecture using web services. A mobile component was also developed to enable ubiquitous access to medical information. This work is based on the authors' knowledge about the Portuguese National Health Service.
\end{abstract}

Keywords: Health information, interoperability, ubiquity, SOA, web services.

\section{Introduction}

The healthcare area is extremely complex due to the new means of diagnostic and therapeutic, new procedures as well as the existence of various professional groups, each one with different characteristics, requirements and ways of working. All of this affects the way information systems in healthcare are implemented and used (Lammintakanen et al, 2010).

For a better decision-making process, especially in a critical area as healthcare, fast and reliable access of a patient's medical history is of utmost importance (Maass et al, 2008; Revere et al, 2007), even though the information is located in a different information system geographically distant from the information system in use in a given situation.

There is an urgent need for the creation of integration mechanisms among the different health information systems that exist today in different healthcare units, like what happens in other areas of activity (Manpaa et al, 2009).

A connection exists between the quality of care provided to a patient and the exchange of information among different levels of healthcare (Maass et al, 2008), like between primary care and differentiated care in the Portuguese National Health Service.

According to the European Commission (2008), the interoperability between health

Copyright (C) 2011 Gonçalo Jorge Rodrigues Plácido, Carlos Rompante Cunha and Elisabete Paulo Morais. This is an open access article distributed under the Creative Commons Attribution License unported 3.0, which permits unrestricted use, distribution, and reproduction in any medium, provided that original work is properly cited. Contact author: Gonçalo Jorge Rodrigues Plácido E-mail: goncalojrplacido@gmail.com 
information systems is of utmost importance and essential for a better health service management, public health, quality and safety of care to patients and clinical research.

Ubiquitous computing possesses the potential for improving the quality of care provided by health professionals outside health institutions (Liu et al, 2011) and facilitates a patient's data collection wherever he may be (Garde et al, 2007).

Information systems in use today are generally proprietary, may be different from health institution to health institution and where designed for local access (Costa et al, 2011; Dogac et al, 2006) which makes heterogeneity, of the existing systems, the main obstacle for the construction of mechanisms to promote interoperability (Pirnejad et al, 2008).

A patient's health information may be spread over an unspecified number of health institutions. (Dogac et al, 2006). For the integration of different systems to be achieved, it is necessary to maintain the integrity of the data between the different systems and to integrate data from more than one source (Pirnejad et al, 2008).

Information systems designed to work on a local level bring challenges at the time of creating an interoperability solution, which technologies to use, what are the human and financial resources needed, how to maintain the integrity of the information, how to maintain the data confidential and private while in transit, among others (Manpaa et al, 2009).

The lack of interoperability between the different systems, which is critical and necessary (Orgun and $\mathrm{Vu}, 2006$ ), leads to redundant, disorganized, disjointed and inaccessible medical information, that may affect the quality of care provided to patients (Raghupathi and Umar, 2008; Li et al, 2001).
Thinking in a different perspective from the one in use today, where the information is centred in healthcare institutions, along side the rise of mobile devices (ANACOM, 2010) and the growing in capacity and functionality of this devices, it makes sense to think about utilizing this kind of devices as auxiliaries in provisioning medical care, distribution of medical information and as an extension to today's systems (Su and $\mathrm{Wu}, 2011$; Sneha and Varshney, 2009; Rigby, 2007).

According to Haux (2006a), traditional institution-centered architectures are not adequate and should be replaced to approaches more patient-centered through 'transinstitutional' information system architectures and infrastructures. This vision is the main stream of this paper.

The objectives of the present work are: study the importance of interoperability and ubiquity of medical information and to propose an architecture based in services using web services; provide interoperability among the different health information systems that exist today and use mobile devices to provide ubiquitous access to the information.

\section{Problem Description}

The Portuguese healthcare system is divided into two main areas: primary care, represented by health centers, and differentiated care, represented by hospitals and other healthcare institutions.

Primary care is the primary source of care and the patients should consult them first, except in cases of emergency. This type of care focuses primarily on general care and proximity with the community, routine consults, monitoring and follow up of chronic patients through the patient's life.

The access to differentiated care is usually performed by the intervention of primary care health professionals or in case of 
emergency. This type of care is more specialized than those in primary care and they are used when the primary care can't give an adequate response in specific situations like surgery or oncology patients (Portuguese Legislation no 56/79, 15th of September).

The health information systems that are used in Portugal were not designed with the objective of being interoperable (Espanha and Fonseca, 2010).

The benefits of the adoption of health information systems by the health institutions are undeniable (Liu et al, 2010; Raghupathi and Umar, 2008; Haux, 2006a). However, the medical information is isolated in the various health institutions (Orgun and $\mathrm{Vu}, 2006)$. This, in fact, has been the usual approach in the implementation of health information systems (Wright and Sittig, 2008).

A patient may consult several healthcare institutions, primary or differentiated, throughout his life, with the creation of medical information in those situations.

The healthcare professional in a hospital is unable to access the information created in a health center. As a rule, a patient starts to take medication by indication of the primary care physician, but when the patient needs to go to a hospital, for whatever reason, the hospital's health professional doesn't know if the patient is on any medication and what is the medication, unless the patient informs him. The opposite also happens, when a patient is submitted to surgery in a hospital, the primary care professional cannot access the information created during the hospitalization nor during surgery, the primary care professional only takes knowledge of what happened when the patient passes him the information in a future consult in the health center.

In emergency situations in which it is extremely important that all relevant medical information about the patient, e.g. pathology, medication, preformed surgeries, is available, the information is not available despite the advantages that the ubiquity of information brings, especially in health related areas (Su and $\mathrm{Wu}, 2011$; Jen et al, 2007; Rigby, 2007; Haux, 2006b).

The medical information created in a hospital is not accessible from a health center and vice-versa. In a given situation, unless the patient is conscious and can give the information that the health professional needs to preform a knowledgeable and personalized decision in the best course of action at that time, the health professional does not have access to the totality of the patient's medical information.

The healthcare professional, to make an informed decision about what procedures to follow, needs access to that information scattered through the different institutions (Wright and Sittig, 2008; Orgun and Vu, 2006; Li et al, 2001).

If in an institution an error by absence of information occurs, depending on the severity, it may be reverted or minimized because the health professional has more resources to his disposal. But if an error by absence of information occurs away from an institution, by a mobile team of the emergency service, where the resources are scarce, reverting the problem will be more difficult if not impossible; hence the need for ubiquitous access to patient's health information (Haux, 2006a).

\section{State of the Art}

To promote interoperability among heterogeneous health information systems and the inclusion of mobile devices in health care, several approaches have been followed.

A model using service-oriented architecture (SOA) and web services was proposed to promote interoperability among different health information systems. The proposed model possesses two different approaches: a procedural approach and a documental 
approach. The procedural approach uses web services to promote the integration of applications using specifications such as Simple Object Access Protocol (SOAP), Web services Definition Language (WSDL) and Universal Description, Discovery and Integration (UDDI). The documental approach tries to describe in detail the elements of the exchange of information among the different systems (Mykkanen et al, 2007).

Service-oriented Architecture for NHIN Decision Support (SANDS) is an architecture based on services to support clinical decision involving services that provide patient data, healthcare information systems and services that make inferences in support to medical information. This architecture contemplates the situation where the medical history of a patient is distributed among different institutions and that to make an inference about a specific situation, more than one system has to be consulted (Wright and Sittig, 2008).

The Total Health Enriching Mobile U-health Service System (THE-MUSS) is a system proposed to the development of ubiquitous solutions to the area of healthcare. The system consists of a Business Process Management System (BPMS), mobile devices, biosensors and a set of primitives defined using web services. These primitives are the base for the development of more complex services. In this system the biosensors communicate with the mobile device using a personal area network (PAN) and the mobile device communicates with the servers using the appropriate service (Han et al, 2010).

The iCabiNET project has the objective to monitor medication intake in outpatients. The system consists in a mobile application, or an application that can be installed in a set-top box, the application communicates with a server, where the health information system is running, using web services Representational State Transfer (REST), SOAP or Java Remote Method Invocation (RMI). The communication is bidirectional, the system warns the patient when necessary to take a medication and what medication; the application confirms the medication's intake or not. The mobile and set-top box applications communicate with each other via Bluetooth or the Internet. The system also makes the identification of the medication to be taken using Radio Frequency IDentification devices (RFID) present in boxes of medicines (Nores et al, 2011).

The Sensor Assistance for Vital Events (senSAVE) is a project developed with the objective of remote monitoring the vital parameters of patients with 50 years of age and older focusing especially on the treatment of cardiovascular diseases. The system consists of a piece of clothing with electrodes in it connected to a portable electrocardiogram machine, a heart frequency-measuring device and oxygen saturation in the blood. These devices transmit the collected data to a Personal Digital Assistant (PDA) via Bluetooth and the PDA communicates with a telemedicine system via the Internet or mobile networks. The mobile application in the PDA informs the patient about his/her vital parameters and issues an alert when the values reach a maximum or minimum limit (Lorenz and Oppermann, 2009).

\section{Proposed Architecture}

Currently an architecture based on services using web services is one of the options in use to promote interoperability among heterogeneous information systems (Serbanati et al, 2011; Liu et al, 2009; Wright and Sittig, 2008; Mykkanen et al, 2007).

The technologies used to promote the ubiquity of medical information ranging from the use of agents, for communication in heterogeneous environments connected by communication networks, to the Internet for remote monitoring ( $\mathrm{Su}$ and $\mathrm{Wu}, 2011$; Sneha and Varshney, 2009); web services for the creation of basic services that serve as basis for the creation of more complex services (Han et al, 2010); ZigBee for communication 
with biosensors (Lee et al, 2009); infrared for communication between devices (Michalowski et al, 2003); 802.11 networks for communicating with a server using an ADSL connection for documenting patient care by health professionals (Chau and Turner, 2006); cable television systems and 802.11 networks for monitoring vital parameters and create medical information in an ubiquitous form (Lin et al, 2008).

To ensure interoperability among the different systems, an architecture is proposed based on services using web services and specifications as WSDL and SOAP.

The use of web services will also serve to communicate with mobile devices with the Android platform making use of ubiquitous computing.

The Hospital (H), Primary Care (PC) and Medical Emergency (ME) subsystems (Figure 1) represented by the Institution in the data model (Figure 2) represent the hospitals, health centers and medical emergency respectively. The GLOBAL subsystem is where the patient can access his/her medical and personal information.
The subsystems of the different institutions replicate the information in their databases with the GLOBAL subsystem database on a daily basis.

In each subsystem, a central component, where the application logic and user interface are located, serves as a bridge between the database and the layer of available services.

The health professional, namely emergency medical, may quickly access all of the patient's information, present in GLOBAL, through a mobile device using the access interface for mobile devices (WSM). To update the ME subsystem, one must access the services provided by the health professional.

The GLOBAL subsystem will consist of a multilayer application with the creation of an access interface for mobile devices using web services.

If the $\mathrm{H}, \mathrm{PC}$ and $\mathrm{ME}$ subsystems, for whatever reason, can't access each other services, they can access the services available in GLOBAL, thus they can get the information they need.

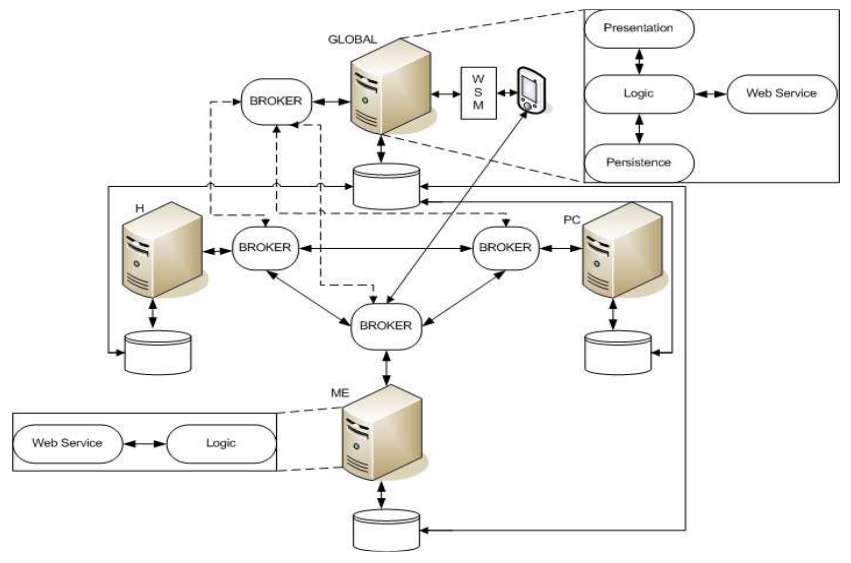

Fig 1. Proposed Architecture

The focus of this architecture is to study a way to promote interoperability between heterogeneous systems, show the shortcomings of the systems in use today and the changes that need to be made to these same systems. 
The H, CS and ME subsystems already exist, and were not modified, to promote interoperability between them. Another piece was created, the BROKER. The BROKER encapsulates the interface and its operations that a subsystem uses to obtain information from another subsystem. The BROKER facilitates the creation of another type of applications in the future, without the need to worry about the internal implementation of the different subsystems.

In the implementation of the prototype, the proposed architecture provides interoperability in three specific cases, consultations in the CS subsystem, surgery/hospitalization in the $\mathrm{H}$ subsystem, and an emergency situation outside a health institution in the EM subsystem. For example, if a patient was submitted to a surgery, the medical information created during that procedure will be stored in subsystem $\mathrm{H}$ and will be accessible to the other subsystems through the operations provided by the BROKER. The same happens with the information stored in the other subsystems.
The proposed mobile component is something, to the best of our knowledge, that does not exist today and is a valuable addition to improve the quality of care, prevent mistakes and provide the most relevant information about a patient outside health institutions, as explained in section 2 .

In the proposed architecture, the patient has easy access to his medical information through the GLOBAL subsystem.

With the proposed architecture, the health information systems can interoperate and enable and provide health information to be accessed wherever and whenever needed.

For the implementation of the prototype of the proposed architecture (Figure 1), a data model (Figure 2) was created that tries to represent a part of the relevant medical information of a patient. This model will be placed in the subsystem GLOBAL (Figure 1) and will be the repository of all information in the proposed architecture. It will also function as a security copy of the patient's information.

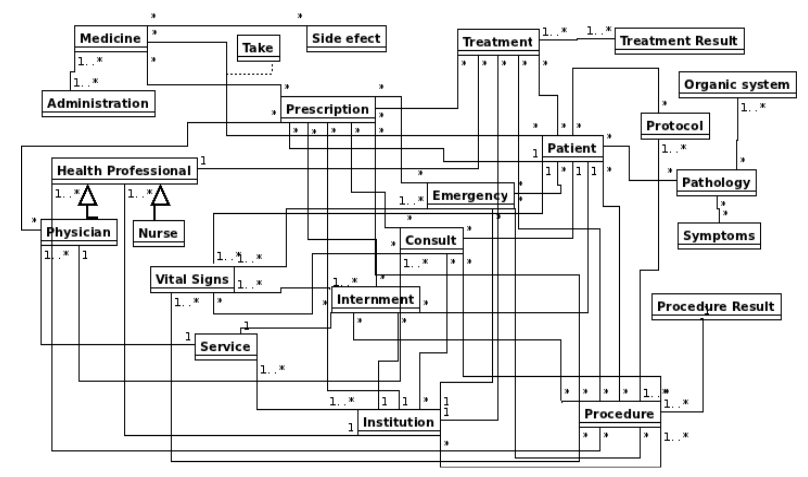

Fig 2. A Reduced Data Model Overview

The proposed model is a possible representation of the organization of medical information, it was designed to implement the prototype and it does not attempt to represent all information that is needed to keep about a patient, yet manages to show the complexity associated with a health information system, even in a small scale as the one used in this work.

Some classes have been left out due to the lack of space, mainly inheritance relations, but that does not affect the global design of the model. 
In this type of systems, it is extremely important to know when a given decision was taken, who took it, the decision affects who, who operationalized the decision and the result of that decision, in order to help determine liability when a mistake is made, which further increases the complexity associated with these systems.

Another reason, health information systems became so complex is the increase of diagnostic and therapeutic procedures and the necessity to store and process the data they produce (Haux, 2006a).

\section{Prototype}

In the implementation of the prototype, interoperability was provided in three specific cases: consultations in the CS subsystem, surgery/hospitalization in the $\mathrm{H}$ subsystem and an emergency situation outside a health institution in the EM subsystem.

The focus of the prototype implementation was the BROKER in the $\mathrm{H}, \mathrm{PC}$ and $\mathrm{ME}$ subsystems and in the communication between them as well as the access to the GLOBAL and ME subsystems using a mobile device.

To promote interoperability between the different subsystems, specifications such as WSDL and SOAP were used.

The proposed data model (Figure 2) was divided among three data repositories, one for the different subsystems, PC, ME and $\mathrm{H}$ presented in the proposed architecture (Figure 1). The GLOBAL subsystem possesses the totality of the model.

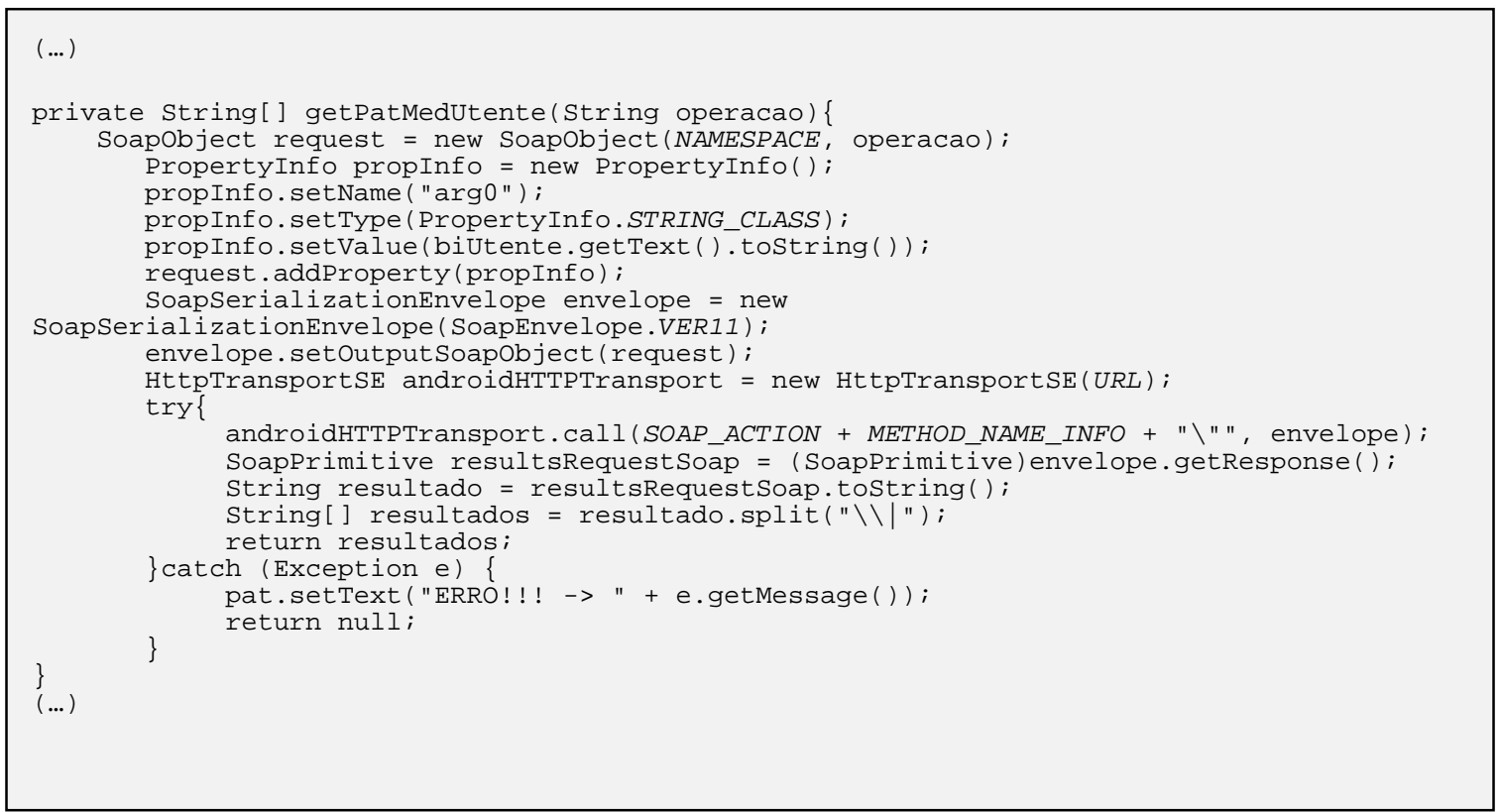

\section{Listing 1 - Method GetPatMedUtente}

Listing 1 shows the event that occurs when a button is pressed in the developed Android application, the device accesses the web service GlobalMobile present in the GLOBAL $\begin{array}{llr}\begin{array}{l}\text { subsystems and invokes one of two } \\ \text { operations, }\end{array} & \begin{array}{l}\text { getPatologias } \\ \text { or }\end{array}\end{array}$ getMedicamentos. 


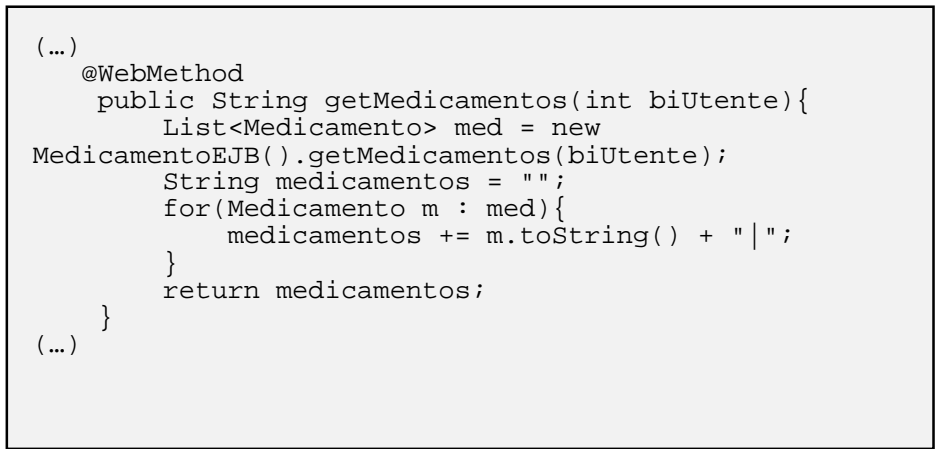

\section{Listing 2 - Web Method Getmedicamentos}

The web method used by the piece of code in Listing 1 is shown in Listing 2. This operation is part of the web service GlobalMobile and allows knowing the medication prescribed to a patient providing the number of the identity card of the same.

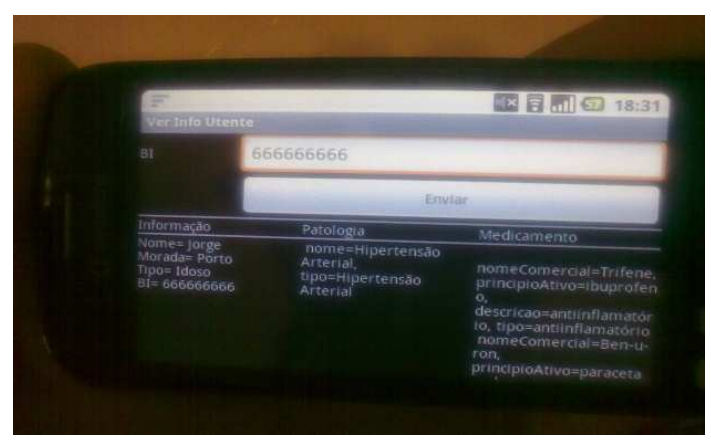

Fig 3. Get Patient's Information

Figure 3 shows the Android application user interface that uses the code in Listing 1. After introducing the patient's identity card number, the GLOBAL subsystem is consulted using the operations defined in the GlobalMobile web service. 


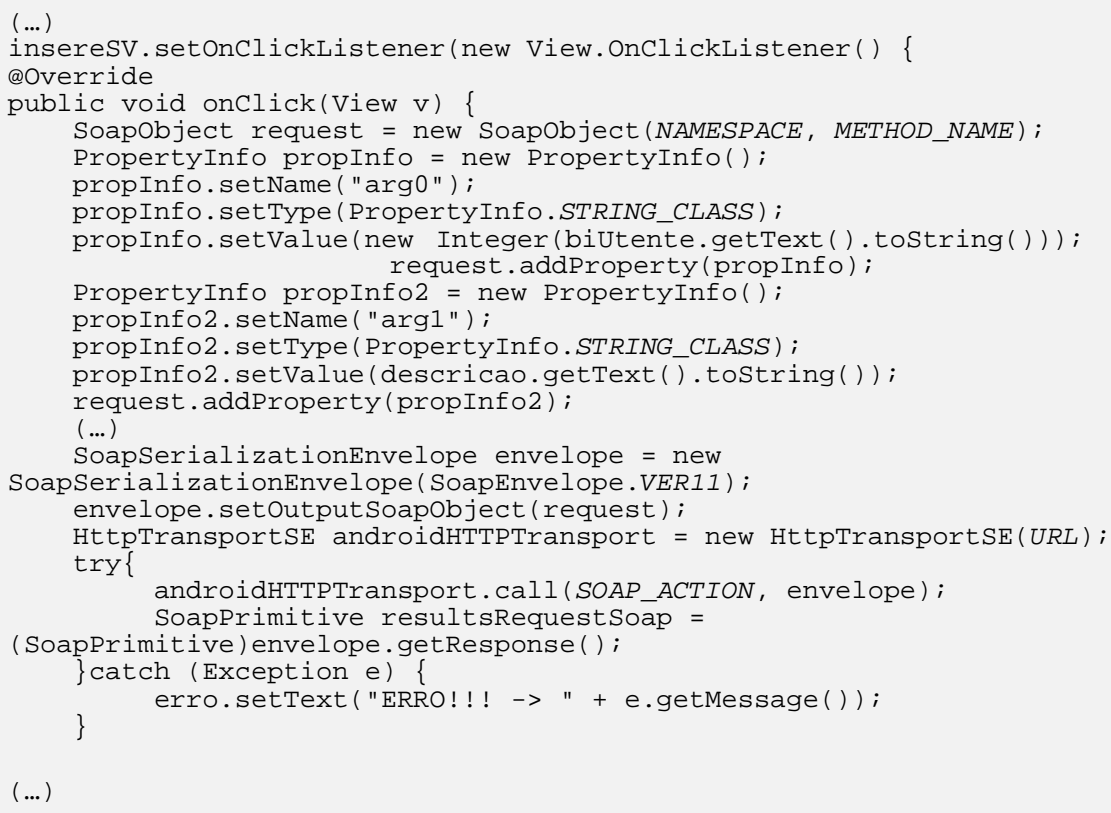

(...)

\section{Listing 3 - Creation of the SOAP Envelope in the Mobile Application}

The Android application (Listing 3) invokes the operation insertSVUrgencia (Listing 4) present in the EMMobile web service in the
EM subsystem passing the parameters presented in figure 4.

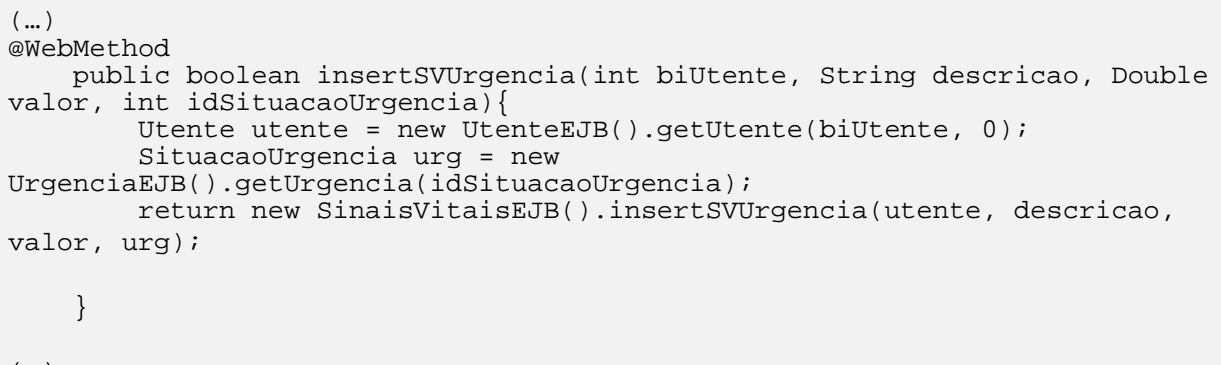

Listing 4 - Web Method Insertsvurgencia

Listing 4 shows the code of the operation insertSVUrgencia provided by the EMMobile web service that allows the insertion of a

\author{
patient's vital parameters in a given \\ emergency situation.
}




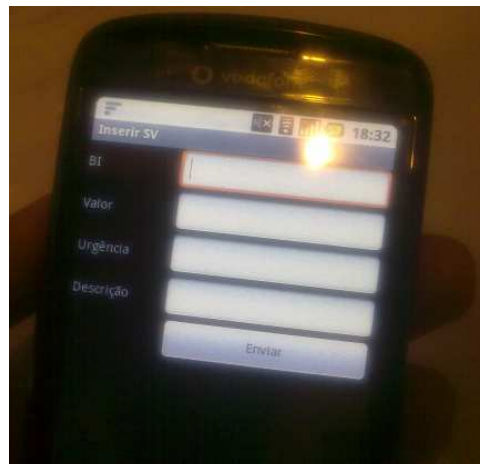

Fig 4. Insertsvurgencia UI Form

Figures 4 and 5 represent the user interface for some of the operations provided by the EMMobile web service in the EM subsystem, namely the insertion of vital parameters (Listing 3) and prescriptions.

Due to the lack of space, is only shown the mobile application and its communication with the subsystems EM and GLOBAL because, to the best of our knowledge, the possibility to access medical information outside health institutions using mobile devices of easy access, does not exist in the Portuguese health system.

\section{Discussion}

Interoperability between the information systems in the different levels of care provides information that is organized, nonredundant and easily accessible, leading to a cut in healthcare costs (Raghupathi and Umar, 2008).

If there is interoperability between the different systems, the patient may have better medical care, because in case he needs it outside his area of residence, his medical information is available.

The care provided will be personalized, adequate to the patient's needs (Haux, 2006a) and not a generalist solution that is not always the best.

Through better management and coordination of medical information, an

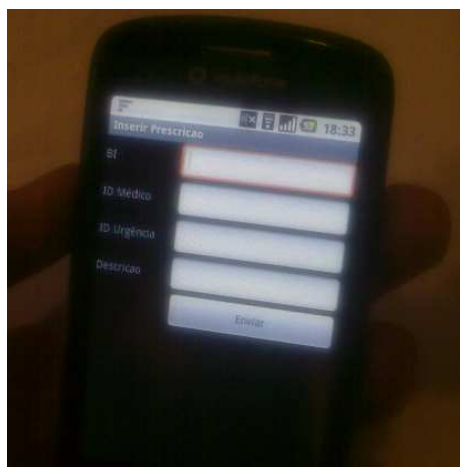

Fig 5. Insertprescricao UI Form

increase in the quality and safety of medical care is provided to the patient (Liu et al, 2010).

Interoperability biggest advantage in a critical and important area as healthcare is increased information available in one of the most critical times if not the most critique and the decision making process (Cho et al, 2010). The bigger and better the amount of information in a given situation, the better the care provided by health professionals and the lower the number of errors (Manpaa et al, 2009).

Interoperability provides that medical information of a patient, by making it easily available to the health professional so that, the latter may take an informed decision about the best medical care for that patient.

Financial benefits are also provided by the interoperability of different health information systems (Jha et al, 2008; Raghupathi and Umar, 2008). less paper is spent; use of media widely available, like the Internet and mobile devices; rationalization of medical and therapeutically procedures, e.g. less duplication of tests; rationalization of medicine prescription, e.g. decrease of double prescription, also leading to a reduction in the cost by the patient.

The ubiquitous access to medical information also brings advantages. It makes possible the remote monitoring of patients, which can mean shorter hospitalization time with 
consequent reduction of costs. It in addition enables a health professional of a mobile unit of the emergency service to have access to medical information outside health institutions ( $\mathrm{Su}$ and $\mathrm{Wu}, 2011$; Sneha and Varshney, 2009).

Challenges need to be overcome when different systems interoperate and make use of ubiquitous computing for accessing the information, especially in healthcare where the information is private and very sensitive.

Commonly, the main obstacle is the heterogeneity of the involved systems, the technologies used, how the data models in the different systems are defined, the age of the systems, just to name a few.

Another challenge associated to the creation of interoperable health information systems is the representation of medical information that is very technical and complex (Raghupathi and Umar, 2008) so that the different systems may understand one another, e.g. how to represent the result of a procedure, or how to represent a given medical situation or pathology. One solution is the use of recommendations/standards such as the CEN/ISO EN13606, Health Level 7 (HL7) and the International Classification of Diseases (ICD) (Nores et al, 2011; Wright and Sittig, 2008).

Other important challenges are: knowing if the information is reliable, if it comes from the right place, who can view it, if the information is delivered to those who requested it, which security policies are created and applied (Gritzalis and Lambrinoudakis, 2004).

The contextualization of the information that the system tries to obtain is another challenge. How to formally define in which context a given information, is more important than another. How to filter all the information available is another problem in the creation of interoperable systems.
Another problem is the use of mobile devices to access private and sensible information, such as medical information. The device must have hardware and software capabilities to support the security requirements needed to access the different systems, e.g. encryption protocols.

To implement complex business systems, like medical information systems (Liu et al, 2009) and in order to enhance what was said earlier, one option to consider is an SOA.

An SOA may be seen as a collection of services, based on interfaces (Wright and Sittig, 2008) that communicate with each other using communication networks, e.g. the Internet, with the goal of coordinating an activity. The services provided by SOA may be implemented using different technologies, e.g. web services (Mykkanen et al, 2007).

Web services are software components that make use of open technologies, such as eXtensible Markup Language (XML) and the Internet, using messages to communicate with each other (Mykkanen et al, 2007). Web services may be utilized to encapsulate the implementation of the different health information systems and expose pre-existing resources (Dogac et al, 2006).

Due to their portability, translated in the use of open technologies associated with supporting programming languages, development and production environments, web services have had an increasing importance in the development of SOAs (Mykkanen et al, 2007). This makes web services one of the best choices to promote interoperability among heterogeneous information systems, like health information systems.

With the proposed architecture, the different health information systems could interoperate and the information can be accessed whenever and wherever needed. 


\section{Conclusion}

Medical information systems are extremely complex and for a better healthcare assistance, on the different levels of care, they should not be isolated and they must support decision making in emergency situations.

This work presents a proposal to promote interoperability among heterogeneous health information systems using a service-oriented architecture and web services. The architecture also enables the ubiquitous access using mobile devices and web services with the objective of providing a better medical assistance inside and outside health institutions.

SOA and web services are a viable option to provide interoperability among heterogeneous information systems, and they possess the capacity of concealing the complexity of those systems exposing only what is necessary.

Promoting interoperability between information systems is so complex and that which deals with such sensible information, as health information systems do, is something that takes a considerable amount of time and involves, a not so small multidisciplinary team.

Another way to potentiate a stricter relationship between patients and health services is to use what today is of relative easy access mobile devices; namely, cellular phones and/or smartphones. With the constant rise of capabilities of these devices be it in processing power, amount of internal memory or the ability to connect to the Internet or other devices, they may be helpful in the remote monitoring of chronic patients, or assistance in emergency situations where the device can save some of the patient's medical information that the health professional can consult and use. Making use of these true handheld computers, it would be possible to improve a patient's quality of life.

\section{References}

ANACOM, (2010), 'Serviços Móveis, Relatório Técnico,' Autoridade Nacional de Comunicações. [Online], [Retrived August 20, 2011], http://www.anacom.pt/render.jsp

Chau, S. \& Turner, P. (2006). "Utilisation of Mobile Handheld Devices for Care Management at an Australian Aged Care Facility',"Electronic Commerce Research and Applications, 5, 305-312.

Cho, I., Kim, J., Kim, J. H., Kim, H. Y. \& Kim, Y. (2010). "Design and Implementation of a Standards-based Interoperable Clinical Decision Support Architecture in the Context of the Korean HER," International Journal of Medical Informatics, 79, 611-622.

Costa, C. M., Menárguez-Tortosa, M. \& Fernández-Breis, J. T. (2011). "Clinical Data Interoperability Based on Archetype Transformation," Journal of Biomedical Informatics.

Dogac, A., Laleci, G. B., Kirbas, S., Kabak, Y., Sinir, S. S., Yildiz, A. \& Gurcan, Y. (2006). "Artemis: Deploying Semantically Enriched Web Services in the Heathcare Domain," Information Systems, 31, 321-339.

Espanha, R. \& Fonseca, R., (2010). 'Plano Nacional de Saúde 2011-2016,' Alto Comissariado da Saúde. [Online], [Retrieved August 20, 2011], http://www.acs.min-saude.pt

European Commission (2008), 'Recomendação da Comissão Relativa à Interoperabilidade Transfronteiriça de Registos de Cuidados de Saúde Electrónicos,' Jornal Oficial da Comissão Europeia. [Online], [Retrieved September 8, 2011], http://eurlex.europa.eu/LexUriServ/LexUriServ.do?uri =CELEX:32008H0594:EN:NOT/

Garde, S., Hovenga, E., Buck, J. \& Knaup, P. (2007). "Expressing Clinical Data with openEHR Archetypes: A Solid Basis for 
Ubiquitous Computing, "International Journal of Medical Informatics, 76S, S334-S341.

Gritzalis, D. \& Lambrinoudakis, C. (2004). "A Security Architecture for Interconnecting Health Information Systems," International Journal of Medical Informatics, 73, 305-309.

Han, D., Lee, M. \& Park, S. (2010). "THEMUSS: Mobile U-Health Service System," Computer Methods and Programs in Biomedicine, 97(2), 178-188.

Haux, R. (2006). "Health Information Systems - Past, Present, Future," International Journal of Medical Informatics, 75, 268-281.

Haux, R. (2006). "Individualization, Globalization and Health - About Sustainable Information Technologies and the Aim of Medical Informatics," International Journal of Medical Informatics, 75, 795-808.

Jen, W.- Y., Chao, C.- C., Hung, M.- C., Li, Y.- C. \& Chi, Y. P. (2007). "Mobile Information and Communication in the Hospital Outpatient Service',"International Journal of Medical Informatics, 76, 565-574.

Jha, A. K., Doolan, D., Grandt, D., Scott, T. \& Bates, D. W. (2008). "The Use of Health Information Technology in Seven Nations," International Journal of Medical Informatics, 77, 848-854.

Lammintakanen, J., Saranto, K. \& Kivinen, T. (2010). "Use of Electronic Information Systems in Nursing Management," International Journal of Medical Informatics, 79, pp. 324-331.

Lee, H. J., Lee, S. H., Ha, K.- S., Jang, H. C., Chung, W.- Y., Kim, J. Y., Chang, Y.- S. \& Yoo, D. H. (2009). "Ubiquitous Health Care Service using Zigbee and Mobile Phone for Elderly Patients," International Journal of Medical Informatics, 78, 193-198.

Lin, C.- C., Lee, R.- G. \& Hsiao, C.- C. (2008). "A Pervasive Health Monitoring Service System Based on Ubiquitous Network
Technology,"International Journal of Medical Informatic, 77, 461-469.

Liu, C., Zhu, Q., Holroyd, K. A. \& Seng, E. K. (2011). "Status and Trends of Mobile-Health Applications for iOS Devices: A Developer's Perspective," The Journal of Systems and Software.

Liu, V., Franco, L., Caelli, W., May, L. \& Sahana, T. (2009). "Open and Trusted Information Systems/Health Informatics Access Control (OTHIS/HIAC)," Proceedings of 7th Australasin Information Security Conference. Wellington, New Zealand, 99-108.

Li, Y. C., Kuo, H. S., Jian, W. S., Tang, D. D., Liu, C. T., Liu, L., Hsu, C. Y., Tan, Y. K. \& Hu, C. H. (2001). "Building a Generic Architecture for Medical Information Exchange among Healthcare Providers," International Journal of Medical Informatics, 61(2), 241-246.

López-Nores, M., Blanco-Fernández, Y., Pazos-Arias, J. J. \& García-Duque, J. (2011). "The iCabiNET System: Harnessing Electronic Health Record Standards from Domestic and Mobile Devices to Support Better Medication Adherence," Computer Standards \& Interfaces.

Lorenz, A. \& Oppermann, R. (2009). "Mobile Health Monitoring for the Elderly: Designing for Diversity," Pervasive and Mobile Computing, 5, 478-495.

Maass, M. C., Asikainen, P., Maenpaa, T., Wanne, O. \& Suominen T. (2008). "Usefulness of a Regional Care Information System in Primary Care a Case Study," Computer Methods and Programs in Biomedicine, 91, 175-181.

Manpaa, T., Suominen, T., Asikainen, P., Maass, M. \& Rostila, I. (2009). "The Outcomes of Regional Healthcare Information Systems in Health Care: A Review of the Research Literature," International Journal of Medical Informatics, 78, 757-771. 
Michalowski, W., Rubin, S., Slowinski, R. \& Wilk, S. (2003). "Mobile Clinical Support System for Pediatric Emergencies," Decision Support Systems, 3, 161-176.

Mykkanen, J., Riekkinen, A., Sormunen, M., Karhunen, H. \& Laitinen, P. (2007). "Designing Web Services in Health Information Systems: From Process to Application Level," International Journal of Medical Informatics, 76(2-3), 89-95.

Orgun, B. \& Vu, J. (2006). "HL7 Ontology and Mobile Agents for Interoperability in Heterogenous Medical Infomation Systems," Computers in Biology and Medicine, 36(2-3), 817-836.

Pirnejad, H., Bal, R. \& Berg, M. (2008). "Building an Inter-organizational Communication Network, and Challenges for Preserving Interoperability," International Journal of Medical Informatics, 77, 818-827.

Raghupathi, W. \& Umar. A. (2008). "Exploring a Model-Driven Architecture (MDA) Approach to Health Care Information Systems Development," International Journal of Medical Informatics, 77(5), 305-314.

Revere, D., Turner, A. M., Madhavan, A., Rambo, N., Bugni, P. F., Kimball, A. \& Fuller, S. S. (2007). "Understanding the Information Needs of Public Health Practitioners: A Literature Review to Inform Design of an Interactive Digital Knowledge Management System," Journal of Biomedical Informatics, 40, 410-421.

Rigby, M. (2007). "Applying Emergent Ubiquitous Technologies in Health: The Need to Respond to new Challenges of Opportunity, Expectation, and Responsibility," International Journal of Medical Informatics, 76, 349-352.

Serbanati, L. D., Ricci, F. L., Mercurio, G. \& Vasilateanu, A. (2011). "Steps Towards a Digital Health Ecosystem," Journal on Biomedical Informatics.
Sneha, S. \& Varshney, U. (2009). "Enabling Ubiquitous Patient Monitoring: Model, Decision Protocols, Opportunities and Challanges,"Decision Support Systems, 46, 606-619.

$\mathrm{Su}$, C.- J. \& Wu, C.- Y. (2011). "JADE Implemented Mobile Multi-Agent Based, Distributed Information Platform for Pervasive Health Care Monitoring," Applied Soft Computing, 11(1), 315-325.

Wright, A. \& Sittig, D. F. (2008). "SANDS: A Service-Oriented Architecture for Clinical Decision Support in a National Information Network',"Journal of Biomedical Informatics, 41(5), 962-981. 